\title{
Perspectives and strategies for anti-doping analysis
}

\author{
Michele Protti ${ }^{1}$, Roberto Mandrioli ${ }^{2}$ \& Laura Mercolini ${ }^{*}, 1$ \\ ${ }^{1}$ Department of Pharmacy \& Biotechnology (FaBiT), Alma Mater Studiorum - University of Bologna, Bologna, Italy \\ ${ }^{2}$ Department for Life Quality Studies (QuVi), Alma Mater Studiorum - University of Bologna, Rimini, Italy \\ *Author for correspondence: Tel./Fax: +39 051 2099726; laura.mercolini@unibo.it
${ }^{6}$ In order to investigate the constantly evolving illicit doping practices in sports, direct screening and confirmation strategies should be continuously updated"

First draft submitted: 1 November 2018; Accepted for publication: 8 November 2018; Published online: 21 January 2019

Keywords: anti-doping • automation • bioanalysis • blood • microsampling • miniaturized biological samples - urine

\section{Bioanalysis in modern anti-doping testing}

Current doping testing in sports provides identification, often followed by quantitation, of banned substances and methods from biological samples collected from athletes in- and out-of-competition. The compounds (approximately 250) and methods included into the World Anti-Doping Agency (WADA) list of prohibited substances and methods [1] as part of the World Anti-Doping Code [2] are classified in the S0-S9 and M1-M3 categories, respectively, while alcohol (P1) and $\beta$-blockers (P2) are forbidden in selected sports. Compounds are divided into non-threshold substances, for which identification is sufficient to provide an adverse analytical finding (AAF), and threshold substances prohibited above a certain level. To harmonize the results obtained from WADA-accredited laboratories, minimum required performance levels for analytical methods have been established, defining the minimum sensitivity requirements for the analysis of non-threshold substances [3]. Urine, and in some cases also hematic matrices (whole blood, serum, plasma), are considered the biological specimens of choice for routine anti-doping analysis. Advantages of urine samples include non-invasive collection and relatively large volumes available, whereas blood collection is invasive and yields a limited volume. Mainly for these reasons, most anti-doping analyses are still performed in urine, even if the prevalence of blood testing is recently increasing. Thus, investigations on the presence of a banned compound in urine are routinely carried out by means of a common workflow, starting with an initial screening followed by a confirmation procedure. The screening step should be fast, selective and sensitive enough to avoid false-negative and false-positive results. In the case of a positive screening result, it must be confirmed by targeting the identified compound and its metabolites [4]. To achieve this workflow on such a wide range of compounds included in the WADA prohibited list, accredited anti-doping laboratories should use multiple analytical methodologies and first of all MS-based ones (GC-MS/MS and LC-MS/MS), considered reference methods due to their higher selectivity and sensitivity. In order to investigate the constantly evolving illicit doping practices in sports, direct screening and confirmation strategies should be continuously updated, by including the monitoring of additional substances with potential doping properties and new metabolites of known compounds with increased detection windows; by taking into account alternative biological matrices to obtain complementary data; by optimizing all the pre-analytical steps to enhance sample stability and maximize result soundness and reliability.

\section{Challenges \& possible solutions in anti-doping analytical research}

From these premises, it is clear that the challenges faced by anti-doping activities are manifold, ranging from political to technical, economical and scientific.

Analytical methods, biological specimens, collection containers and pretreatment procedures are continually updated and made tighter and more sophisticated in response to ever-increasing sophistication in doping illicit 
practices in sports. This vicious circle leads to increasing costs and in turn to limiting anti-doping procedures to a tiny fraction of the sporting community, leaving out most amateurs and 'minor' sports [5]. One of the first unmet needs of the anti-doping community is to have analytical methods and practices able to provide reliable information, but also faster and cheaper.

Reliable analytical results are the most important factor: no effective action can be undertaken unless sound analytical data is available. Constant progress in instrumentation efficacy, procedure reliability and scientific knowledge of doping agents are all factors that certainly contribute to better data quality. Miniaturization and automation are two of the possible approaches for both reducing expenses and increasing reliability. Sample miniaturization can make both sampling and analysis less expensive through reduced use of materials and timeintensive treatment procedures and can increase sample stability, thus leading to more reliable results $[6,7]$. A wealth of new microsampling and miniaturized pretreatment techniques are currently available and some of these are probably already mature for implementation into anti-doping procedures: dried blood spots (DBS) have been studied for more than 50 years and can be used with remarkably reliable results in many cases, if the procedures are adequately validated. Other microsampling and miniaturized pretreatment procedures are still less well-known, but increasingly included in bioanalytical method development. For example, volumetric absorptive microsampling (VAMS) [8], on-line micro solid phase extraction ( $\mu$ SPE) [9], microextraction by packed sorbent (MEPS) [10], as well as the direct coupling between microsamples and analytical platforms, are promising and cutting-edge bioanalytical approaches [11]. The strategies that use dried microsamples in particular, such as dried matrix spots (DMS) and VAMS, can enhance sample stability also related to transportation and long-term storage, which can be key factors for the success of anti-doping analysis. Microsampling also allows detection of analytes, even when the specimen/matrix is scarce or even minimal, granting new possibilities and expanding the range of applicability of most anti-doping analytical methods. Automation, on the other hand, is increasingly being applied in highthroughput sample pretreatment and analysis, since it promises to greatly reduce human sources of error, while multiplying productivity and reducing costs. While automation is surely very attractive, its wider application is currently still held back, mainly by a lack of appropriate knowledge, especially in the field of proper instrumentation validation and also by very strict regulation in the field.

Better knowledge about possible and current doping substances is also needed. If commercial therapeutic agents, such as many anabolic steroids, $\beta$-agonists, erythropoietin (EPO) analogs, are well-known under many aspects (pharmacokinetics, mechanism of action, side effects, toxicity), there is an increasing number of new psychoactive substances, and by extension 'new doping substances', currently being introduced onto illegal markets. Very little is known about these active compounds. Miniaturization and automation can play a very positive role in expanding scientific knowledge on new chemical entities [12], whatever their intended use. In fact, in view of the 3Rs principles (replacement, reduction and refinement) [13], microsampling can have a big impact according to two of the three principles: it reduces the need to sacrifice laboratory animals and also allows for the possibility of using the same animals for several different experiments by enhancing their welfare (refining).

Identifying new doping agents in a timely manner is a long-standing challenge. Both drugs of abuse (which are prohibited in-competition) $[14,15]$ and compounds used specifically for doping purposes are the subject of intense, illegal study to produce new entities; for example, new 'designer steroids' are constantly synthesized and then used 'on the field' for doping purposes [16]. Non-targeted bioanalytical approaches using advanced MS techniques (including high-resolution mass spectrometry and specifically tailored structure libraries and databases) are probably the best bet toward finding as yet unknown compounds [17].

Other challenges include the recent use of short-lived and endogenous substances for doping purposes, the main example being synthetic or natural peptides and hormones. Regarding exogenous peptides, their detection is made particularly difficult by the very low concentrations involved, their intrinsic instability and their relatively nonspecific metabolites. In this field too, dried matrix microsampling techniques are very promising, in the perspective of increasing analyte stability and thus detectability [18].

When endogenous peptides are involved, accurate isotopic abundance evaluation techniques are frequently used. Other possibilities include the use of a biological passport and the detection of appropriate markers, for example in the case of growth hormone, several proteins, peptides and propeptides have been proposed as candidate markers [19]. However, this problem remains decidedly still open.

Finally, microdosing seems to be becoming the cutting edge in doping practices, especially for steroids and EPO [20]. Using very low, frequent doses seems to allow obtaining performance increases while making detection ever more difficult. The most promising bioanalytical answer to this practice is probably the use of increasingly 
sensitive strategies. Alternative matrices (for example, hair) could be useful and also advantageous if accumulation can be demonstrated.

\section{Conclusion}

Bioanalysis is continually evolving and reaching new goals. Within this unending process, anti-doping analysis follows its own trajectory, but cannot go forward alone and has to use all of the bioanalytical strategies and tools, adapting them to sport testing. Among these, biological matrix choice is one of the most important weapons. Samples alternative to blood and urine have to be considered, since they can provide complementary information, or specifically accumulate some substances. Dried miniaturized matrices have substantial advantages over wet ones, such as a generally better stability at room temperature and consequently more favorable behavior during storage and transportation. For these reasons, dried microsampling and MS analysis represent probably the best prospect to become one of the golden standards at the forefront of anti-doping bioanalysis research. Coupling this setup with sample pretreatment and analysis automation and miniaturization could produce important progress in this field.

\section{Financial \& competing interests disclosure}

The authors have no relevant affiliations or financial involvement with any organization or entity with a financial interest in or financial conflict with the subject matter or materials discussed in the manuscript. This includes employment, consultancies, honoraria, stock ownership or options, expert testimony, grants or patents received or pending, or royalties.

No writing assistance was utilized in the production of this manuscript.

\section{References}

1 World Anti-Doping Agency. Prohibited

list (2019). https://www.wada-ama.org/en/resources/science-medicine/prohibited-list-documents

2 World Anti-Doping Agency. World anti-doping

code (2018). https://www.wada-ama.org/en/resources/the-code/world-anti-doping-code

3 World Anti-Doping Agency. Technical document TD2018MRPL: minimum required performance levels for detection of prohibited substances (2018). https://www.wada-ama.org/en/resources/science-medicine/td2018mrpl-0

4 World Anti-Doping Agency. International standard for laboratories (2016). https://www.wada-ama.org/en/resources/laboratories/international-standard-for-laboratories-isl

5 Outram SM, Stewart B. Condemning and condoning: elite amateur cyclists' perspectives on drug use and professional cycling. Int. J. Drug Policy 26(7), 682-687 (2015).

6 Protti M, Catapano MC, Samolsky Dekel BG et al. Determination of oxycodone and its major metabolites in haematic and urinary matrices: comparison of traditional and miniaturised sampling approaches. J. Pharm. Biomed. Anal. 152, 204-214 (2018).

7 Saracino MA, Catapano MC, Iezzi R, Somaini L, Gerra G, Mercolini L. Analysis of $\gamma$-hydroxy butyrate by combining capillary electrophoresis-indirect detection and wall dynamic coating: application to dried matrices. Anal. Bioanal. Chem. 407(29), 8893-8901 (2015).

8 Protti M, Mandrioli R, Mercolini L. Tutorial: volumetric absorptive microsampling (VAMS). Anal. Chim. Acta 1046, 32-47 ( 2019).

9 Verplaetse R, Henion J. Quantitative determination of opioids in whole blood using fully automated dried blood spot desorption coupled to on-line SPE-LC-MS/MS. Drug Test. Anal. 8(1), 30-38 (2016).

10 Mercolini L, Mandrioli R, Protti M, Conca A, Albers LJ, Raggi MA. Dried blood spot testing: a novel approach for the therapeutic drug monitoring of ziprasidone-treated patients. Bioanalysis 6(11), 1487-1495 (2014).

11 Hecht M, Evard H, Takkis K et al. Sponge spray - reaching new dimensions of direct sampling and analysis by MS. Anal. Chem. 89(21), 11592-11597 (2017).

12 Mercolini L, Protti M. Biosampling strategies for emerging drugs of abuse: towards the future of toxicological and forensic analysis. $J$. Pharm. Biomed. Anal. 130, 202-219 (2016).

13 Törnqvist E, Annas A, Granath B, Jalkesten E, Cotgreave I, Öberg M. Strategic focus on 3R principles reveals major reductions in the use of animals in pharmaceutical toxicity testing. PLoS ONE 9(7), e101638 (2014).

14 Protti M, Rudge J, Sberna AE, Gerra G, Mercolini L. Dried haematic microsamples and LC-MS/MS for the analysis of natural and synthetic cannabinoids. J. Chromatogr. B Analyt. Technol. Biomed. Life Sci. 1044-1045, 77-86 (2017).

15 Mercolini L, Protti M, Catapano MC, Rudge J, Sberna AE. LC-MS/MS and volumetric absorptive microsampling for quantitative bioanalysis of cathinone analogues in dried urine, plasma and oral fluid samples. J. Pharm. Biomed. Anal. 123, 186-194 (2016).

16 Abushareeda W, Fragkaki A, Vonaparti A et al. Advances in the detection of designer steroids in anti-doping. Bioanalysis 6(6), 881-896 (2014). 
17 de Albuquerque Cavalcanti G, Rodrigues LM, dos Santos L et al. Non-targeted acquisition strategy for screening doping compounds based on GC-EI-hybrid quadrupole-Orbitrap mass spectrometry: a focus on exogenous anabolic steroids. Drug Test. Anal. 10(3), 507-517 (2018).

18 Sleczka BG, D’Arienzo CJ, Tymiak AA, Olah TV. Quantitation of therapeutic proteins following direct trypsin digestion of dried blood spot samples and detection by LC-MS-based bioanalytical methods in drug discovery. Bioanalysis 4(1), 29-40 (2012).

19 Tan S-H, Lee A, Pascovici D et al. Plasma biomarker proteins for detection of human growth hormone administration in athletes. Sci. Rep. 7(1), 10039 (2017).

20 Clark B, Woolford SM, Eastwood A, Sharpe K, Barnes PG, Gore CJ. Temporal changes in physiology and haematology in response to high- and micro-doses of recombinant human erythropoietin. Drug Test. Anal. 9(10), 1561-1571 (2017). 